\title{
Évaluation exergetique des changements metaboliques sur l'esperance de vie des fumeurs
}

\section{Exergy assessment of metabolic changes on life expectancy of smokers}

\author{
Izabela B. Henriques ${ }^{1}$, Carlos E. K. Mady², Silvio de Oliveira Junior ${ }^{3}$ \\ ${ }^{1}$ Institut Technologique de l'Aéronautique, São José dos Campos, Brésil, izabela@ita.br \\ 2 École de Génie Méchanique, Université de Campinas, Brésil, cekmady@fem.unicamp.br \\ ${ }^{3}$ École Polytechnique de I'Université de São Paulo, Brésil, soj@usp.br
}

\begin{abstract}
RÉSUMÉ. Quelques auteurs ont déterminé le comportement exergétique du corps humain dans des conditions standard. Certaines de ces études comprennent une estimation de l'espérance de vie basée sur l'association de la création d' entropie / destruction exergétique et la théorie du taux de vie. Le métabolisme est connu comme source d'irreversibilité majeure dans le corps humain. Ainsi, les altérations métaboliques devraient affecter son comportement exergétique et, selon le théorie de taux de la vie, l'espérance de vie. Certains auteurs ont découvert que même les fumeurs sans maladies liées au tabagisme présentent un métabolisme plus élevé que les non-fumeurs, qui contribue à la prise de poids après l'arrêt du tabac. Selon la littérature médicale, le métabolisme des fumeurs est $20 \%$ supérieur à celui des non-fumeurs, en plus de l'effet thermogénique après avoir fumé des cigarettes. Dans le présent travail, l'analyse exergétique est appliquée au corps humain afin d'évaluer l'effet de l'augmentation métabolique chez les fumeurs sur le comportement exergétique et l'espérance de vie. L'analyse prend en compte les taux d'exergie dus à la convection, au rayonnement, à la respiration, à l'évaporation et au métabolisme exergétique. Un modèle thermique à segments multiples du corps humain disponible dans la littérature est utilisé pour effectuer une analyse énergétique. Les résultats de l' exergie détruite intégrée sur la durée de vie indiquent qu'un fumeur détruit environ $730 \mathrm{MJ} / \mathrm{kg}$ d'exergie en plus qu'un non-fumeur jusqu'à l'âge de 73,5 ans, ce qui représenterait une diminution de 15 ans de l'espérance de vie pour ce groupe.

ABSTRACT. A few authors have determined exergy behavior of human body under standard conditions. Some of these studies include estimate of life expectancy based on the association of entropy creation / exergy destruction and rate of living theory. Metabolism is known as the major source of irreversibility in human body. Thus, metabolic alterations are expected to affect its exergy behavior and, according to rate of living theory, life expectancy. Some authors discovered that even smokers without smoking-related diseases present metabolism higher than nonsmokers, which contributes to weight gain after smoking cessation. According to medical literature, metabolism of smokers is $20 \%$ greater than non-smokers, in addition to the known thermogenic effect after cigarette smoking. In the present work, exergy analysis is applied to human body in order to evaluate the effect of metabolic increase in smokers on exergy behavior and life expectancy. The analysis takes into account exergy rates and flow rates due to convection, radiation, respiration, evaporation and exergy metabolism. A multi-segment thermal model of human body available in literature is utilized to perform First Law analysis. Results of destroyed exergy integrated over life span indicate that a smoker destroys around $730 \mathrm{MJ} / \mathrm{kg}$ more exergy than a non-smoker until the age of 73.5 , which would represent a decrease of 15 years in life expectancy for this group.

MOTS-CLÉS. analyse exergétique, métabolisme, tabagisme, espérance de vie.

KEYWORDS. Exergy analysis, metabolism, smoking, life expectancy.
\end{abstract}

\section{Indroduction}

Afin de mieux évaluer la qualité des différents processus de conversion d'énergie, des analyses d'énergie et d'entropie ont été combinées, donnant ainsi le concept d'exergie, qui est une propriété thermodynamique définie comme le travail utile maximum qui peut être obtenu lorsqu'un système est amené en équilibre thermodynamique avec l'environnement au moyen de processus réversibles. Comme indiqué par Szargut [SZA 05], un domaine potentiel d'application de l'analyse exergétique est l'étude des organismes vivants pour estimer l'efficacité exergétique des processus qui se déroulent dans leurs systèmes, organes et cellules. Les premières étapes vers l'analyse exergétique des êtres vivants ont été franchies en appliquant le concept de génération d'entropie. Prigogine \& 
Wiame [PRI 46] ont établi le principe de la production d'entropie minimale, qui définit que les organismes tendent, tout au long de leur vie, à un état de génération d'entropie minimale. Une autre étude associant énergie et vie a été menée par Rubner [RUB 08], qui a été un pionnier dans le développement de la soi-disant Théorie du taux de vie, qui postule que plus le métabolisme est élevé, plus la durée de vie est courte. En comparant le métabolisme énergétique des animaux domestiques et de l'homme, l'auteur a observé que le taux métabolique augmentait en fonction de la taille corporelle pour les mêmes classes vertébrales, c'est-à-dire les mammifères, les reptiles et les amphibiens. Selon lui, la dépense énergétique de $1 \mathrm{~kg}$ de cellules pendant la durée de vie est constante, quelle que soit l'espèce animale où se trouvent ces cellules. En outre, Speakman [SPE 05] a analysé le métabolisme par unité de masse tout au long du cycle de vie de différents mammifères et a conclu que ce paramètre ne varie pas de manière significative entre les différentes espèces. De plus, il a également conclu que, parmi la même espèce, un gramme de tissu dépense approximativement la même quantité d'énergie.

Motivés par le principe de Prigogine et Théorie du taux de vie, certains auteurs ont étudié la génération d'entropie au cours de la vie. Selon Hershey [HER 10], à partir d'une certaine quantité d'énergie libérée par le métabolisme, il n'est plus possible de maintenir la vie. Par conséquent, le corps est proche de l'équilibre thermodynamique avec l'environnement [SCH 92]. De plus, Hershey [HER 10] affirme que la quantité physique plus appropriée pour évaluer la durée de vie est l'entropie, car sa valeur cumulée augmente avec le vieillissement, atteignant son maximum juste avant la mort. Sur cette base, il propose une échelle différente pour évaluer la progression de la vie, appelée âge entropique. En supposant que toute l'entropie générée dans le corps humain est due au métabolisme, il a estimé que l'entropie totale générée pendant la durée de vie serait de $10025 \mathrm{~kJ} / \mathrm{kg}$ $\mathrm{K}$ pour un homme. Une analyse similaire a été réalisée par Silva et Annamalai [SIL 09], en calculant l'entropie générée tout au long de la vie sur la base de l'apport alimentaire, obtenant une valeur de $11404 \mathrm{~kJ} / \mathrm{kg} \mathrm{K}$. Mady et al. [MAD 13a] ont appliqué l'analyse exergétique au corps humain et déterminé l'exergie cumulative détruite de la naissance à 80 ans d'un homme brésilien standard en tenant compte du métabolisme exergétique et des transferts et flux exergétiques vers l'environnement. Pour les conditions basales, l'exergie détruite et l'efficacité exergétique étaient respectivement de $3091 \mathrm{MJ} / \mathrm{kg}$ et 3,5\%, tandis que pour les conditions sédentaires, ces valeurs étaient de $3599 \mathrm{MJ} / \mathrm{kg}$ et $4,3 \%$.

Étant donné que le métabolisme exergétique est responsable de la majeure partie de l'exergie détruite dans le corps [MAD 13b], les conditions physiologiques caractérisées par des altérations du métabolisme ont également tendance à entraîner des modifications de la durée de vie. Dans cet esprit, Henriques et al. [HEN 14] ont étudié l'effet de l'augmentation du métabolisme observée chez les personnes obèses sur le comportement exergétique du corps tout au long de la vie. Ils ont observé que les sujets obèses détruisent plus d'exergie au cours de leur vie par rapport aux personnes maigres. Cependant, en ce qui concerne l'exergie totale détruite par unité de masse, qui aurait une valeur limite au cours de la vie, les valeurs obtenues pour les sujets obèses étaient inférieures à celles des sujets maigres, ce qui contredit les données statistiques sur la mortalité. Ainsi, ils ont conclu que la réduction de l'espérance de vie chez les personnes obèses est principalement liée à une recrudescence de maladies liées à l'obésité, n'ayant rien à voir avec l'augmentation du métabolisme. De plus, en analysant d'autres paramètres exergétiques, ils ont conclu qu'un corps obèse est moins efficace qu'un corps maigre, ce qui indique que l'obésité met en danger le comportement exergétique du corps humain.

Une autre condition qui entraîne des altérations métaboliques est le tabagisme. En tentant d'expliquer la prise de poids observée après l'arrêt du tabac, certains auteurs ont découvert que les fumeurs ont un métabolisme plus élevé que les non-fumeurs. Moffat et Owens[MOF 91] ont mené une expérience qui a présenté des données métaboliques de fumeurs en bonne santé, de nonfumeurs, d'anciens fumeurs et d'anciens fumeurs qui sont retournés fumer. Ils sont parvenus à la 
conclusion que l'augmentation du métabolisme à cause du tabagisme disparaît après l'arrêt du tabagisme. Les valeurs de dépense énergétique obtenues dans cette étude ne tenaient pas compte de l'effet thermogénique du tabagisme qui, selon Kromhout et al. [KRO 88] entraînerait une augmentation journalière supplémentaire de $262 \mathrm{kcal}$ de la dépense énergétique. Sur la base des données expérimentales fournies par ces études, dans le présent travail, l'analyse exergétique est appliquée au corps humain afin de déterminer l'impact de l'augmentation métabolique sur le comportement exergétique global du corps humain. Des modèles énergétiques et exergétiques de la littérature sont utilisés pour effectuer les analyses. À partir des valeurs de l'exergie détruite cumulée, l'impact de l'augmentation métabolique sur l'espérance de vie des fumeurs est déterminé, ainsi que d'autres paramètres exergétiques.

\section{Méthodes}

\subsection{Modèle exergétique}

Mady et al. [MAD 12] ont développé un modèle exergétique du corps humain qui prend en compte non seulement le métabolisme exergétique, mais aussi les interactions exergétiques entre le corps et l'environnement. Le modèle a été divisé en deux volumes de contrôle, le premier (VC1) représentant les systèmes corporels (thermique, circulatoire et respiratoire), tandis que le second (VC2) indique le métabolisme cellulaire, comme on peut le voir sur la Figure 1. L'entrée et la sortie de masse sont supposées égales et les variations quotidiennes de la masse corporelle dues au écoulent et au stockage des substances sont négligées. Les interactions du VC1 avec l'environnement sont provoquées par les flux exergétiques dus à la respiration $\left(\Delta B_{a}\right)$ et à l'évaporation $\left(B_{e}\right)$ et aux transferts exergétiques associés à la convection $\left(B_{c}\right)$ et au rayonnement $\left(B_{r}\right)$. Le modèle comprend également le transfert thermique $Q_{M}$ entre $\mathrm{VC} 1$ et $\mathrm{VC} 2$, qui entraîne un transfert exergétique $B_{Q M}$.

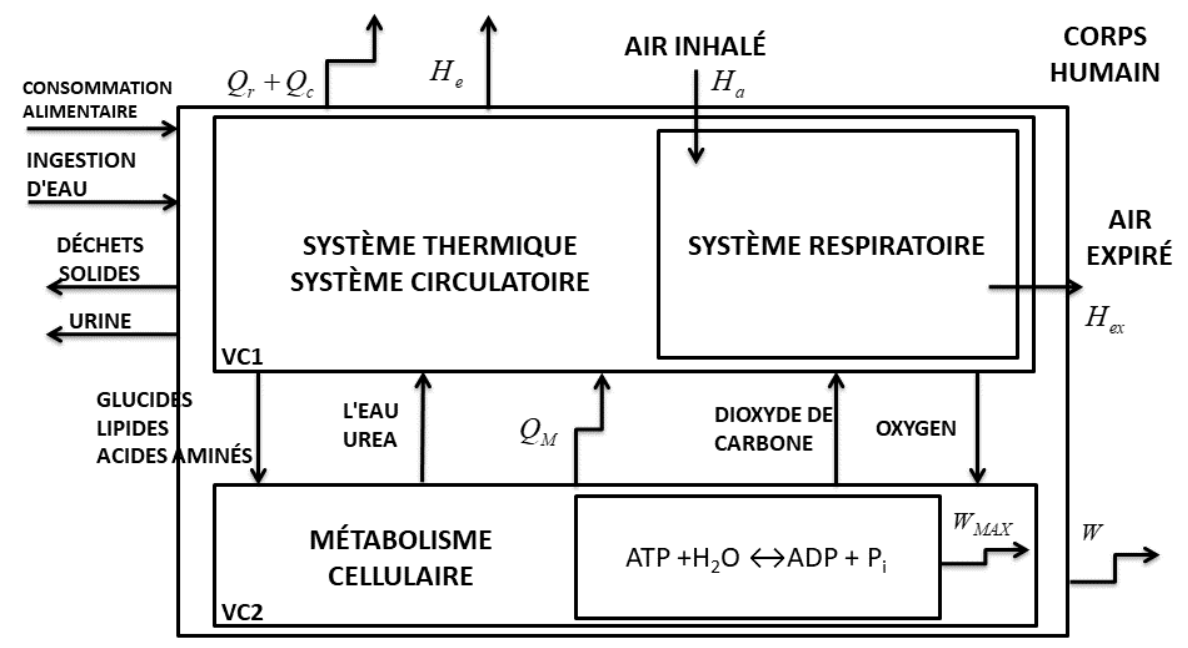

Figure 1. Représentation du corps humain. Adapté de Mady et al. [MAD 12].

En appliquant le bilan exergétique au premier volume de contrôle, on obtient une exergie détruite dans VC1 $\left(B_{d, V C l}\right)$, comme indiqué dans l' Eq. [1], où le dernier terme indique une variation exergétique due à des conditions environnementales transitoires, qui est entièrement attribuée au VC1 :

$$
B_{d, V C l}=B_{Q_{M}}-\left(B_{c}+B_{r}+B_{e}+\Delta B_{a}\right)-\left.\frac{d \mathbf{B}_{\text {corps }}}{d t}\right|_{\Delta T}
$$


Le métabolisme cellulaire, représenté dans le deuxième volume de contrôle, est divisé en deux étapes. Le premier comprend le métabolisme exergétique $\left(B_{M}\right)$, qui est l'oxydation complète des nutriments ingérés, et la formation de molécules d'ATP (adénosine tri-phosphate), qui sont responsables de la libération d'énergie dans le corps pour effectuer le travail, interne ou externe. A partir de l'énergie chimique stockée dans les molécules d'ATP, un travail maximum $W_{M A X}$ peut être effectué. Ce processus rejette la chaleur $\left(Q_{M, A T P}\right)$ et détruit une certaine quantité d'exergie, exprimée en Eq. [2] :

$$
B_{d, A T P}=B_{M}-B_{Q_{M}, A T P}-W_{M A X}
$$

L'ATP produit lors de la première étape du métabolisme cellulaire est hydrolysé lors de la seconde étape, de sorte que le corps est capable d'effectuer un travail à la fois interne $\left(W_{\text {int }}\right)$ et externe $\left(W_{\text {ext }}\right)$. La conversion de l'énergie stockée dans les molécules d'ATP en travail $\left(W=W_{\text {int }}+\right.$ $\left.W_{e x t}\right)$ comprend certaines irréversibilités et rejette également la chaleur $\left(Q_{M}\right.$, hyd $)$. L'exergie détruite dans cette partie du métabolisme cellulaire est :

$$
B_{d, h y d}=W_{M A X}-W-B_{Q_{M}, h y d}
$$

L'exergie totale détruite dans le métabolisme cellulaire $\left(B_{d, V C 2}\right)$ est la somme de l'exergie détruite à chaque étape. Donc,

$$
B_{d, C V 2}=B_{M}-B_{Q_{M}, A T P}-B_{Q_{M}, h y d}-W=B_{M}-B_{Q_{M}}-W
$$

Compte tenu du fait que la chaleur rejetée par le métabolisme est une différence entre le métabolisme et le travail effectué, il convient de noter que plus le travail effectué par rapport au maximum disponible est faible, plus les parcelles $\mathrm{Q}_{\mathrm{M}}$ et $\mathrm{B}_{\mathrm{QM}}$ sont importantes. Enfin, l'exergie détruite dans le corps est la somme de la destruction exergétique dans chaque volume de contrôle. L'addition d' Eq. [1] et Eq. [4] donne:

$$
B_{d, c o r p s}=B_{M}-\left.\frac{d \mathbf{B}_{\text {corps }}}{d t}\right|_{\Delta T}-\left(B_{c}+B_{r}+B_{e}+\Delta B_{\text {air }}\right)-W
$$

Batato et al. [BAT 90] a principalement réalisé l'analyse exergétique du corps humain, et a conclu que le métabolisme énergétique et exergétique ont l'équivalent magnitudes (différence inférieure à $5 \%$ ). Les auteurs ont évalué l'efficacité exergétique, néanmoins, proche de zéro. Mady et OliveiraJunior [MAD 13b] ont déterminé le métabolisme exergétique en fonction des débits massiques de la consommation d'oxygène, de la production de dioxyde de carbone et de l'excrétion d'azote dans l'urine et ont comparé sa valeur au métabolisme sur la base de l'énergie. Les résultats ont montré que la différence entre le métabolisme énergétique et exergétique n'est pas supérieure à 5\%. Par conséquent, dans le présent travail, $B_{M}$ et $M$ sont supposés être égaux. L'exergie totale détruite au cours de la vie $\left(B_{d, a c}\right)$ est déterminée en intégrant $B_{d, \text { corps }}$ sur la durée de vie. De plus, l'efficacité énergétique du corps en l'absence de variations de température ambiante est déterminée comme:

$$
\eta_{\text {corps }}=1-\frac{B_{d, c o r p s}}{B_{M}}
$$

\subsection{Modèle thermique}

Le modèle thermique de Ferreira et Yanagihara [FER 09] fournit les données nécessaires pour réaliser l'analyse de 18 à 80 ans. Comme le montre la Figure 2, le modèle géométrique est composé de 15 cylindres à section elliptique, où chaque segment a sa combinaison de certains tissus 
possibles, à savoir, la peau, la graisse, les muscles, les os, le cerveau, les viscères, les poumons et cœur . De plus, chaque tissu est caractérisé par son propre métabolisme, son débit de perfusion sanguine et ses propriétés thermophysiques. Transfert de chaleur et de masse sont dictées par les systèmes passif et thermorégulation. Les données globales du modèle anatomique sont: hauteur 1,76 $\mathrm{m}$, poids $67 \mathrm{~kg}$, surface $1,8 \mathrm{~m}^{2}$ et volume $0,0627 \mathrm{~m}^{3}$.

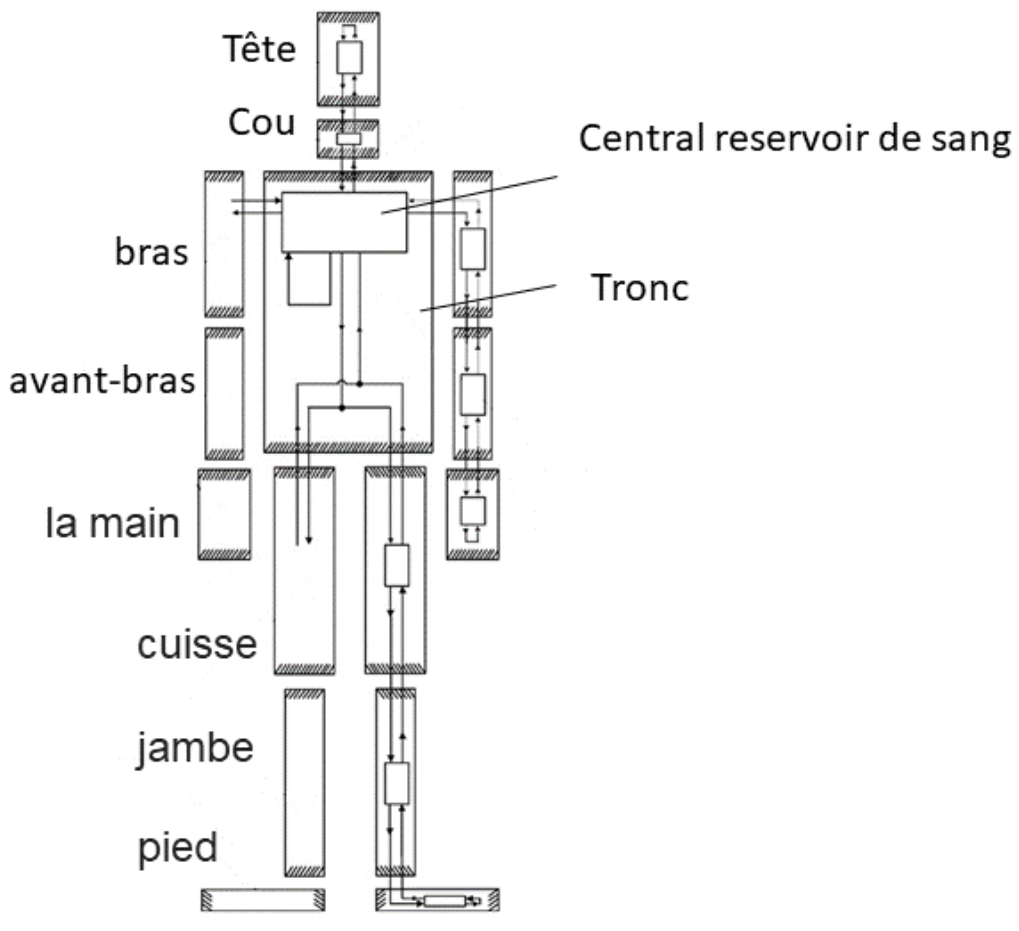

Figure 2. Représentation du modèle thermique développé par Ferreira \& Yanagihara [14].

\subsection{Age exergétique}

Mady et al.[MAD 13a] ont déterminé l'exergie détruite cumulative pour les citoyens brésiliens dans des conditions basales et sédentaires de la naissance à 80 ans sur la base des données anthropométriques disponibles auprès de l'IBGE (Institut brésilien de géographie et de statistiques) [IBG 10]. Leurs résultats, qui sont définis comme référence pour le présent travail, sont présentés dans la Figure 3.

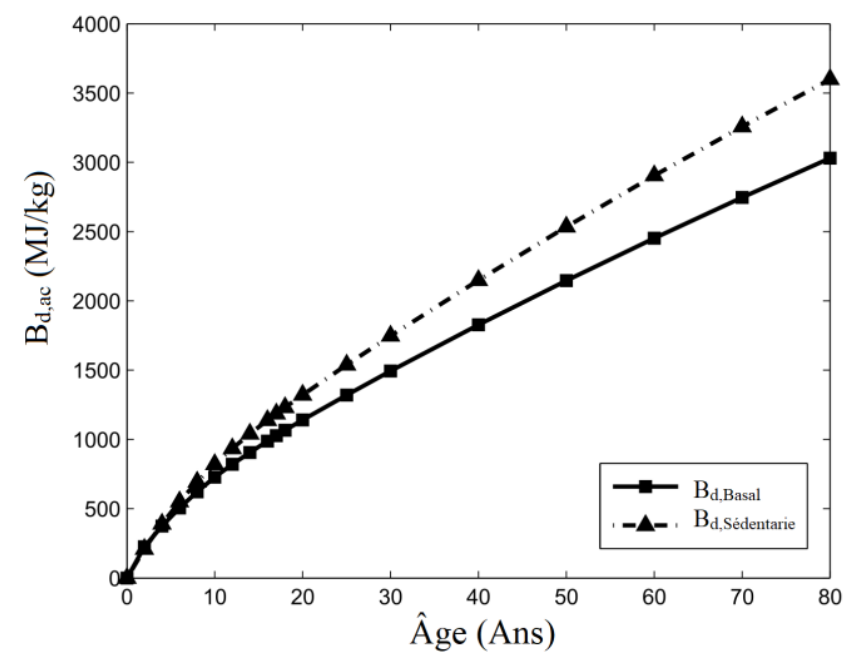

Figure 3: Exergie détruite cumulée pour les citoyens brésiliens [MAD 13a]. 
Selon l'IBGE [IBG 10], l'espérance de vie à la naissance de la société brésilienne est de 73,5 ans. À cet âge, un citoyen brésilien typique accumule une quantité de $3412 \mathrm{MJ} / \mathrm{kg}$ d'exergie détruite. Cette valeur est la référence pour déterminer l'espérance de vie des groupes analysés. En outre, le graphique présenté sur la Figure 3 est la référence pour l'âge exergétique dans la présente étude. L'âge exergétique est supposé être l'âge présenté dans le graphique de la Figure 3 qui correspond à la quantité d'exergie cumulée détruite à un certain âge chronologique pour le sujet analysé.

\subsection{Données d'entrée}

Pour obtenir le bilan énergétique du corps humain de la naissance à 80 ans, Mady et al. [MAD 13a] ont développé un modèle thermique cylindrique du corps humain composé de quatre couches concentriques, à savoir le cœur, les muscles, la graisse et la peau. En raison de sa simplicité, ce modèle est plus adapté à l'analyse du corps humain pendant la phase de croissance, où des changements considérables dans les données anthropométriques sont observés. On suppose que le sujet standard analysé a commencé à fumer à l'âge de 18 ans. Ainsi, les données sur la croissance de la naissance à 17 ans seront supposées égales à celles obtenues par Mady et al.[MAD 12] pour cette période. Ces valeurs seront nécessaires pour déterminer l'exergie détruite cumulée. En raison de sa précision, le modèle thermique décrit dans la section 2.2 est utilisé pour obtenir les paramètres liés au bilan énergétique du corps de 18 à 80 ans, en supposant qu'aucun changement dans la composition corporelle, seulement des variations du métabolisme. Des simulations ont été effectuées pour la neutralité thermique et la température ambiante de $30,4^{\circ} \mathrm{C}$, qui est la température de neutralité thermique obtenue pour le métabolisme le plus bas. Ainsi, il est assuré qu'il n'y a pas d'augmentation du taux métabolique associé au frisson, qui est un mécanisme de régulation thermique activé en dessous pour des températures inférieures à la neutralité thermique.

Moffat et Owens [MOF 91] ont étudié les causes possibles de la prise de poids après l'arrêt du tabac en établissant des différences métaboliques entre les fumeurs, les anciens fumeurs et les nonfumeurs. L'échantillon de fumeurs ne présentait aucune maladie liée au tabagisme et les sujets pouvaient être classés comme gros fumeurs, en raison de la consommation d'au moins 10 cigarettes par jour. Ils ont découvert que, indépendamment de l'effet thermogénique juste après après avoir fumé des cigarettes, les fumeurs auraient un métabolisme par zone superficielle de $20 \%$ supérieur à celui des non-fumeurs. Il convient de souligner que cette étude a été menée auprès d'un groupe de fumeurs qui ne présentaient aucune maladie liée au tabagisme. Ainsi, les variations métaboliques trouvées ne sont liées qu'à l'habitude de fumer, pas à la présence de maladies. De plus, Kromhout et al. [KRO 88] ont trouvé une valeur supplémentaire de $262 \mathrm{kcal} /$ jour associée à l'effet thermogénique du tabagisme. Gardant cela à l'esprit, les valeurs du taux métabolique utilisées par Mady et al. [MAD 13a] pour les non-fumeurs ont été modifiés afin d'obtenir les valeurs correspondantes pour les fumeurs.

\section{Résults et discussion}

Les indices d'exergie le long de la durée de vie obtenus à partir de l'analyse exergétique sont présentés dans les figures 4 à 10. Les figures 4 (a) et (b) illustrent le taux de métabolisme exergétique $\left(B_{M}\right)$ pour les deux groupes et les températures ambiantes. Une augmentation moyenne de 34,6 \% est observée entre fumeurs et non-fumeurs. Plus précisément, à neutralité thermique, une augmentation de $32,8 \%$ et $37,1 \%$ est enregistrée pour les âges de 18 et 80 ans, respectivement. À une température ambiante de $30,4{ }^{\circ} \mathrm{C}$, des valeurs de $34,5 \%$ et $38,4 \%$ sont observés. En comparant les résultats obtenus à la neutralité thermique et à $30,4{ }^{\circ} \mathrm{C}$, on observe que chez les fumeurs $B_{M}$ augmente de $2,1 \%$ à l'âge de 18 ans et seulement $0,1 \%$ à 80 ans. Pendant ce temps, pour les nonfumeurs, ces valeurs ne sont que de $0,8 \%$ et $0 \%$. 
La variation du taux d'exergie détruit $\left(B_{d}\right)$, représentée sur les figures 5 (a) et (b), suit la même tendance observée pour le métabolisme exergétique avec une augmentation légèrement plus petite, d'environ $33,9 \%$. Des augmentations de 32,2\% et 36,7\% ont été observées entre les non-fumeurs et les fumeurs à la neutralité thermique pour 18 et 80 ans, respectivement, tandis que 33,7\% et 36,9\% étaient enregistrés à $30,4^{\circ} \mathrm{C}$. Pour les mêmes âges, les augmentations entre la neutralité thermique et $30,4{ }^{\circ} \mathrm{C}$ sont de $1,2 \%$ et $1,3 \%$ pour les fumeurs et de $0,6 \%$ et 0 pour les non-fumeurs.

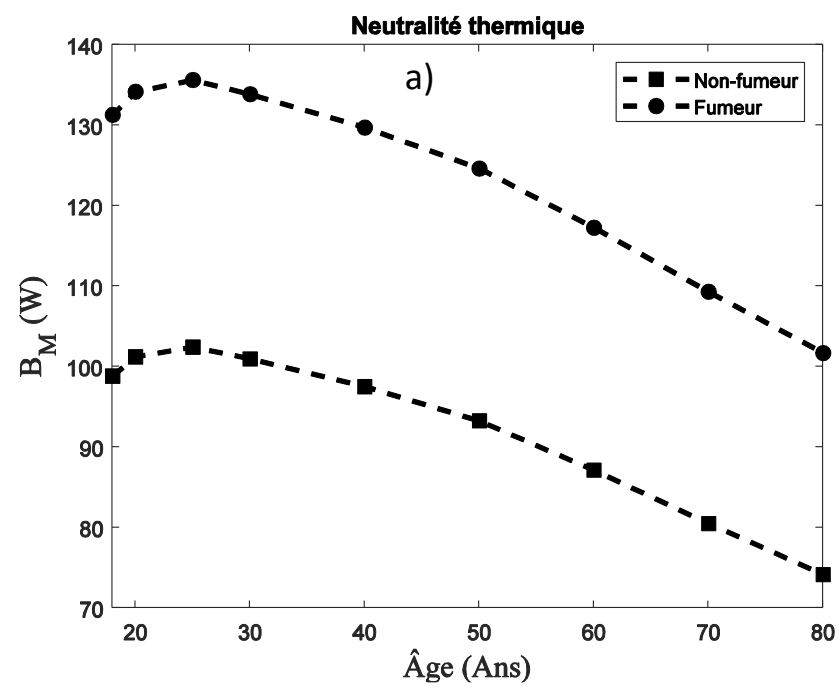

b)

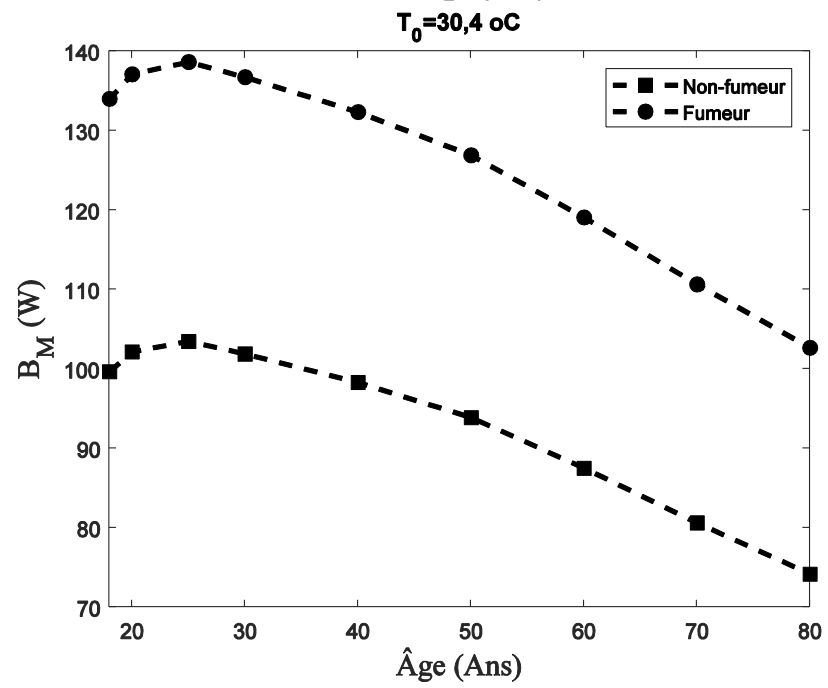

Figure 4. Métabolisme exergétique $\left(B_{M}\right)$ en fonction de l'âge pour les fumeurs et les non-fumeurs, pour la neutralité thermique (a) et $T=30,4^{\circ} \mathrm{C}$ (b). 


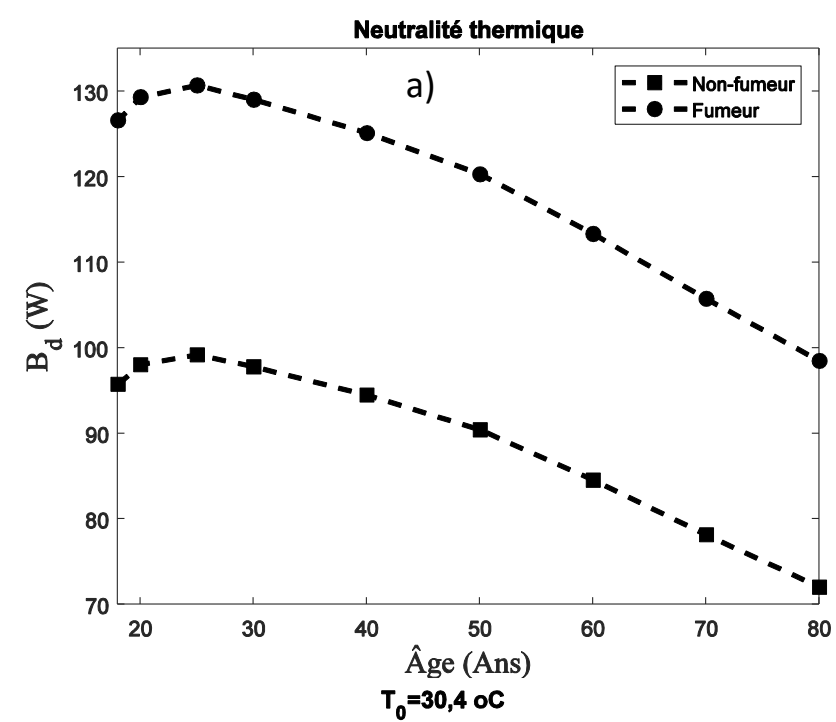

b)

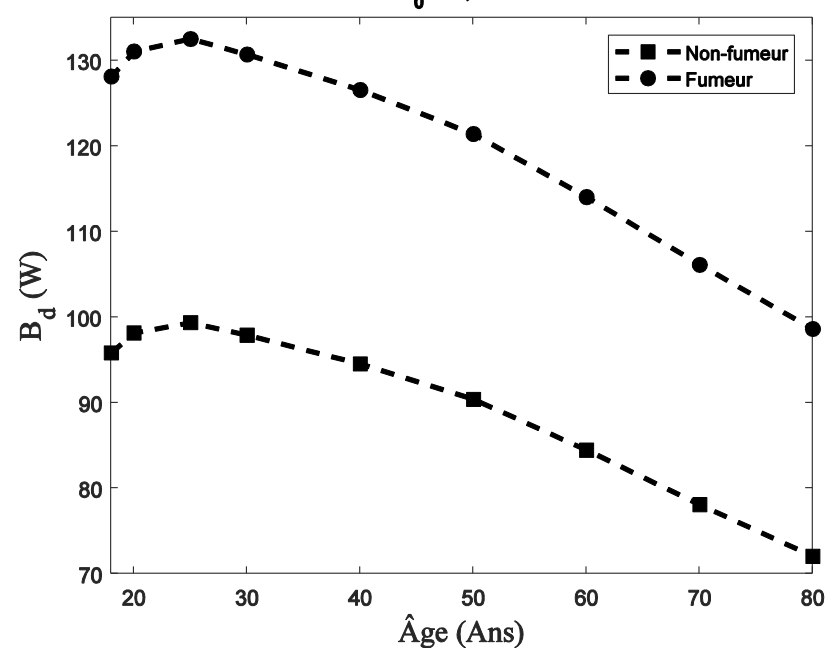

Figure 5. Exergie détruite en fonction de l'âge pour les fumeurs et les non-fumeurs, pour la neutralité thermique (a) et $T=30,4^{\circ} \mathrm{C}$ (b).

La Figure 6 indique les résultats obtenus pour l'efficacité exergétique $\left(\eta_{e x}\right)$. Comme indiqué dans l'équation [6], l'efficacité exergétique, dans des conditions d'état stables, dépend strictement du rapport exergie détruit/métabolisme exergétique. À première vue, il peut sembler que $B_{M}$ et $B_{d}$ varient à peu près dans la même proportion entre les fumeurs et les non-fumeurs. On ne s'attend donc pas à de grandes différences d'efficacité exergétique. Toutefois, lorsque les résultats de l'efficacité exergétique sont analysés, l'impact des petites variations décrites dans les paragraphes précédents devient clair. Pour la neutralité thermique, la variation moyenne de $B_{M}$ est supérieure de $1,8 \%$ à celle observée pour $B_{d}$. Cet accroissement entraîne une augmentation moyenne de $13,8 \%$ de l'efficacité exergétique des fumeurs. Pour $30,4{ }^{\circ} \mathrm{C}$, cet effet est plus prononcé. $B_{M}$ augmente de $2,7 \%$ de plus que $B_{d}$, tandis que l'efficacité exergétique est de $19,1 \%$ supérieure pour les fumeurs dans cette condition. Il est possible de conclure qu'une légère augmentation du métabolisme exergétique par rapport à l'exergie détruite se transforme en un grand avantage pour la valeur d'efficacité exergétique. Par conséquent, étant donné que le pourcentage d'augmentation du taux d'exergie détruit est inférieur à celui observé dans le taux métabolique, le corps d'un fumeur présente une efficacité exergétique plus élevée par rapport à un non-fumeur. 


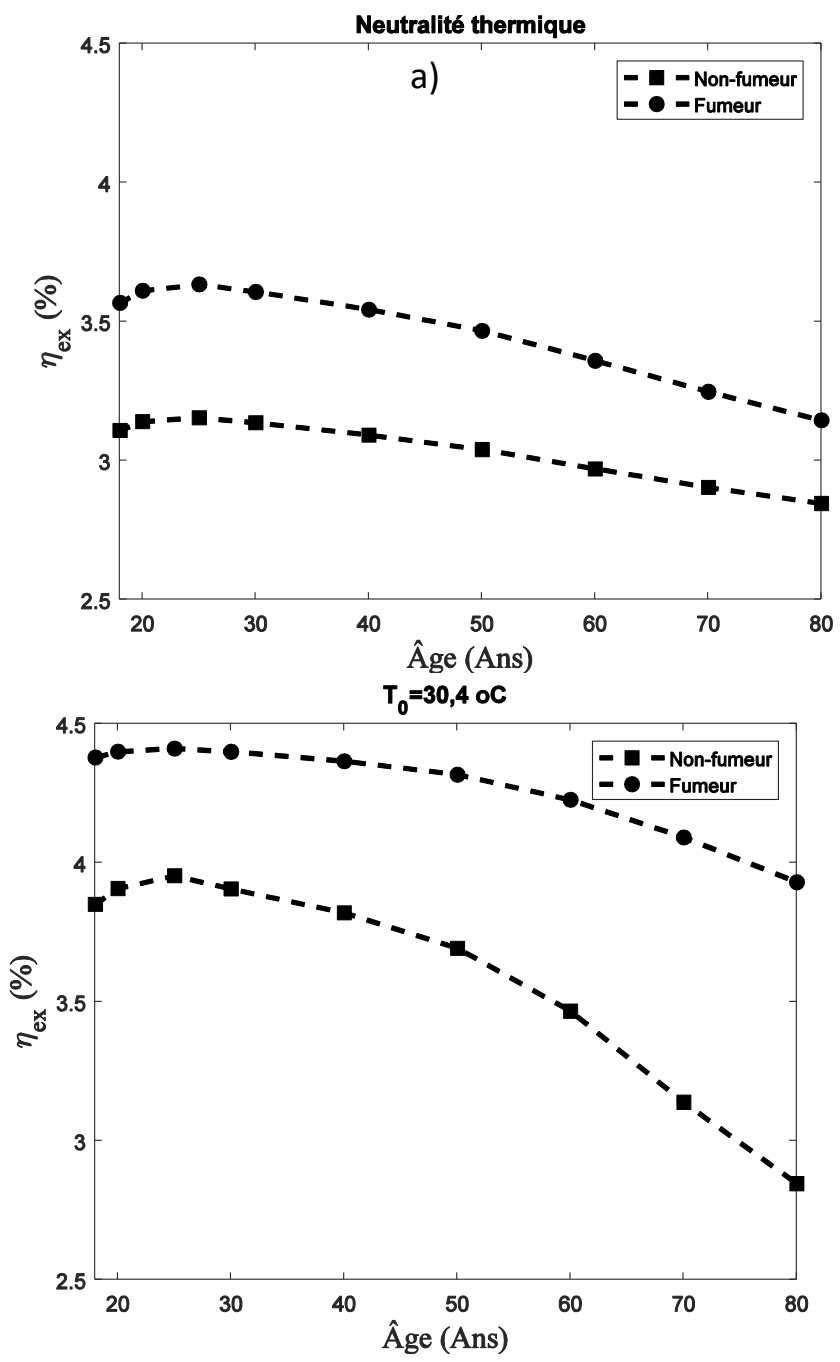

b)

Figure 6. Efficacité exergétique en fonction de l'âge pour les fumeurs et les non-fumeurs, pour la neutralité thermique (a) et $T=30,4^{\circ} \mathrm{C}(\mathrm{b})$.

Un résultat supplémentaire peut être extrait de la Figure 7. Lorsque le sujet est soumis à une température ambiante supérieure à la neutralité thermique, la somme des parcelles exergétiques transférées dans l'environnement $\left(B_{\text {env }}\right)$ augmente. Compte tenu du bilan exergétique, le plus grand transfert d'exergie vers l'environnement conduit à une augmentation de l'exergie détruite inférieure à celle observée pour le métabolisme exergétique, conduisant, enfin, à des rendements exergiques plus élevés hors neutralité thermique. 


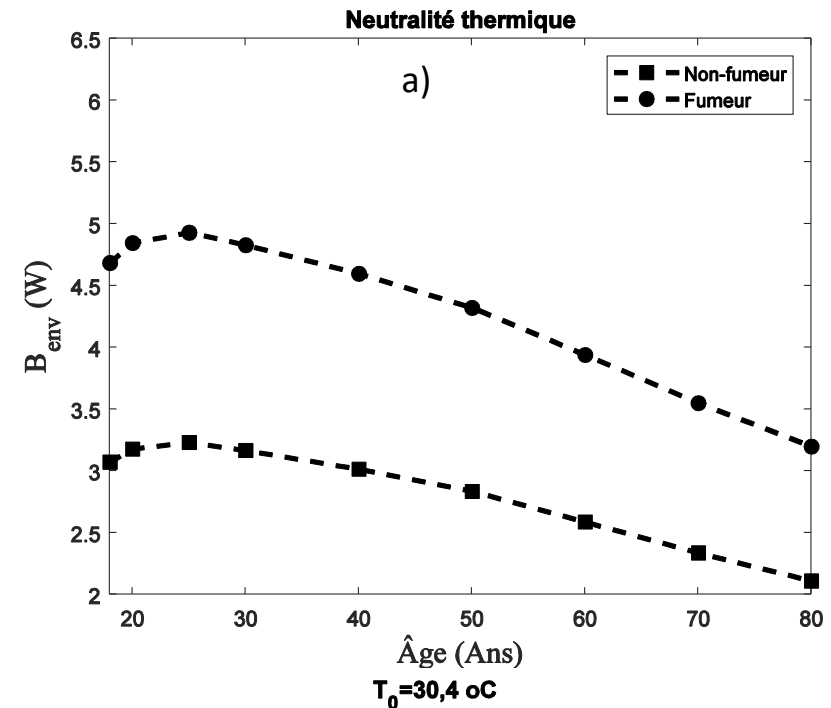

b)

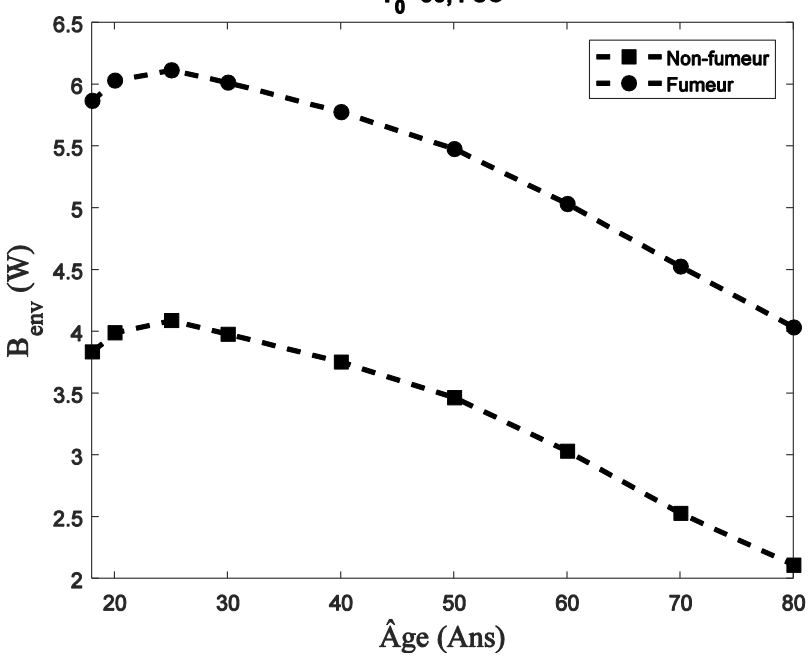

Figure 7. Exergie associée au transfert de chaleur vers l'environnement en fonction de l'âge pour les fumeurs et les non-fumeurs, pour la neutralité thermique (a) et $T=30,4{ }^{\circ} \mathrm{C}$ (b).

Aucune différence significative n'est observée pour l'exergie cumulée détruite par unité de masse $\left(B_{d, a c}\right)$ entre la neutralité thermique et $30,4{ }^{\circ} \mathrm{C}$, comme le montre la Figure 8 . Comme prévu, les fumeurs accumulent plus d'exergie détruite au cours de leur vie. À l'âge de 80 ans, un fumeur aura accumulé, en raison uniquement de l'augmentation métabolique, environ $690 \mathrm{MJ} / \mathrm{kg}$ d'exergie détruite de plus qu'un non-fumeur. Prenant comme référence pour l'espérance de vie la valeur de $3412 \mathrm{MJ} / \mathrm{kg}$, ce qui correspond chronologiquement à l'âge de 73,5 ans, un gros fumeur sans maladies liées au tabagisme perdrait 15,1 ans de vie en raison d'altérations métaboliques. Hors neutralité thermique, cette valeur est supérieure de 4 mois. L'âge exergétique (Figure 9) peut être déterminé en comparant les résultats actuels à ceux obtenus par Mady et al.[MAD 12]. Il est possible d'observer que l'âge exergétique progresse plus rapidement pour les fumeurs, indiquant une réduction de l'espérance de vie. Il faut souligner que les résultats obtenus concernent uniquement l'effet des altérations métaboliques associées au tabagisme sur l'espérance de vie, les sujets ayant participé à la procédure expérimentale n'ayant présenté aucune maladie liée au tabagisme. En tenant compte de ce type de pathologie, la réduction de l'espérance de vie devrait être plus prononcée, mais ce point ne peut être vérifié que par des tests avec des fumeurs atteints d'un certain type de maladie. 


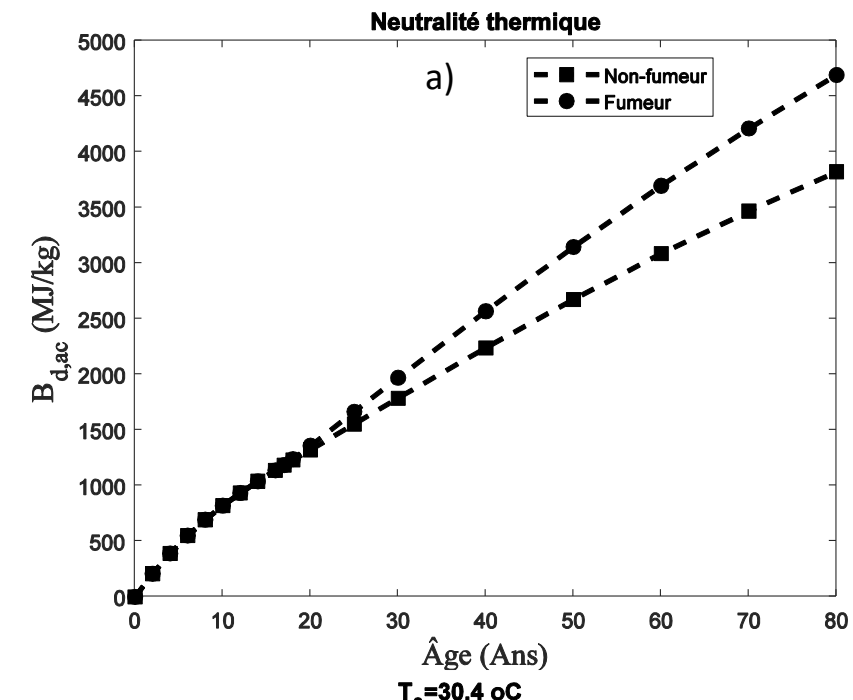

b)

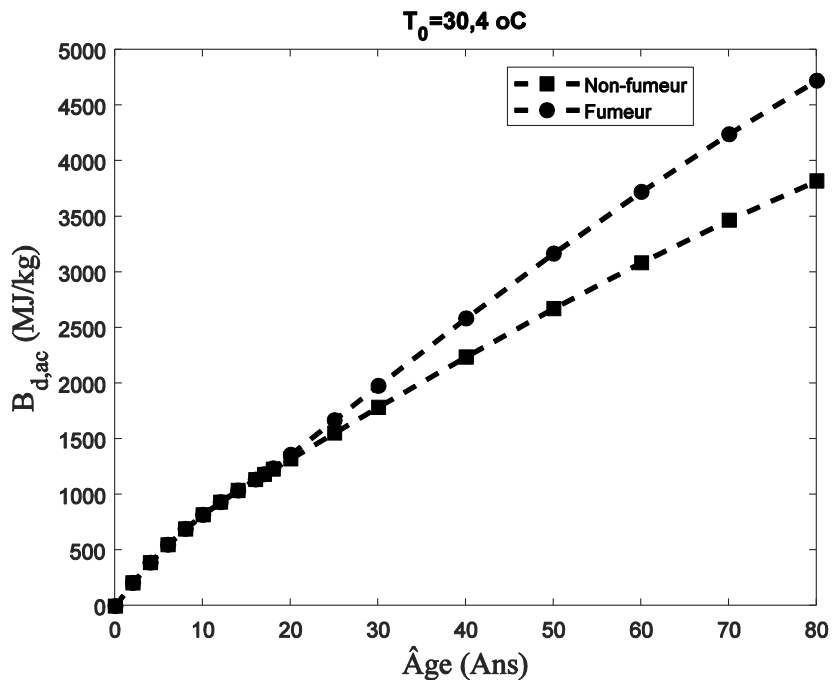

Figure 8. Exergie détruite cumulée en fonction de l'âge pour les fumeurs et les non-fumeurs, pour la neutralité thermique (a) et $T=30,4^{\circ} \mathrm{C}(\mathrm{b})$. 


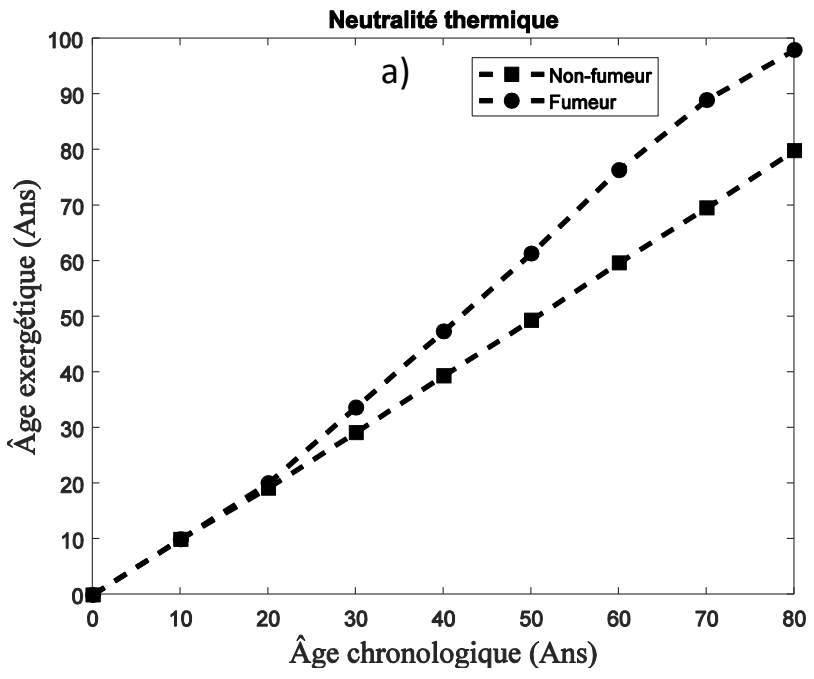

b)

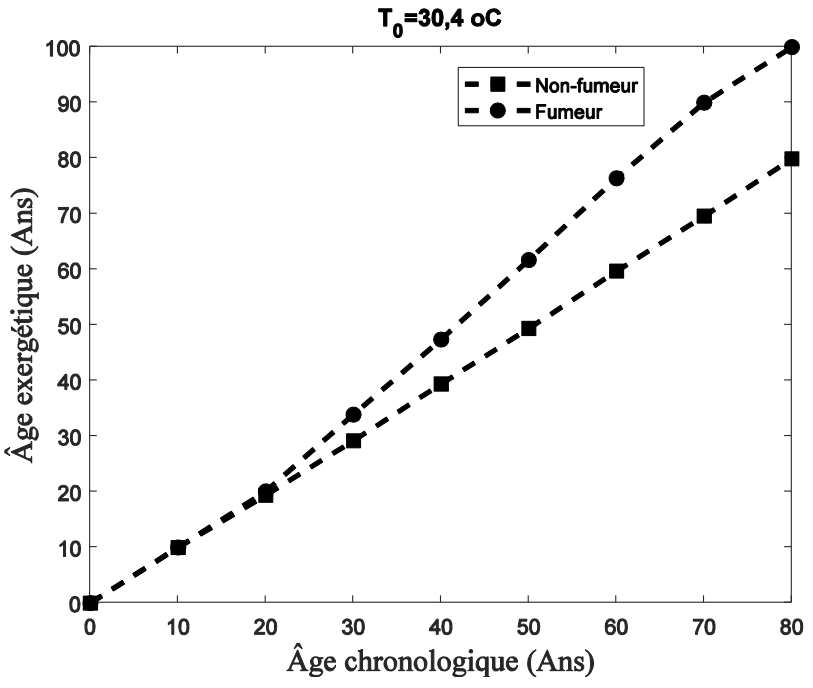

Figure 9. Âge exergétique par rapport à l'âge chronologique pour les fumeurs et les non-fumeurs, pour la neutralité thermique (a) et $T=30,4^{\circ} \mathrm{C}$ (b).

\section{Conclusions}

Une analyse exergétique a été réalisée afin d'évaluer les effets des altérations métaboliques sur l'espérance de vie des fumeurs sans maladies liées au tabagisme. On a observé que le métabolisme exergétique et le taux d'exergie détruit chez les fumeurs suivaient la même tendance à la hausse par rapport aux non-fumeurs, avec une augmentation du métabolisme d'environ $2 \%$ supérieure à celle de l'exergie détruite. Concernant l'efficacité exergétique, c'était le seul paramètre avec des changements pertinents entre les températures de référence évaluées. Dans les deux scénarios, l'efficacité exergétique des fumeurs était plus élevée, en raison de l'augmentation plus élevée observée du métabolisme exergétique par rapport au taux d'exergie détruite. Cependant, cette valeur était encore plus élevée pour la température de $30,4^{\circ} \mathrm{C}$, car la différence entre l'augmentation de $B_{M}$ et $B_{d}$ était plus grande pour cette température. L'origine de cette différence est la somme des flux et transferts exergétiques vers l'environnement, supérieure à la neutralité thermique extérieure. Comme il a été supposé lors de la modélisation qu'aucune variation anthropométrique ne se produit entre les fumeurs et les non-fumeurs, l'exergie détruite accumulée par unité de masse au cours d'une vie était plus élevée pour les fumeurs. Ainsi, l'indicateur d'âge exergétique pour ce groupe progresse plus rapidement que pour les non-fumeurs, indiquant une réduction de l'espérance de vie d'environ 15 ans associée à des altérations métaboliques. 


\section{Bibliographie}

[BAT 90] Batato M., Borel L., Deriaz O., Jequier E., « Analyse exergétique théorique et expérimentale du corps humain», Entropie, n² 26, p.120-130, 1990.

[FER 09] FERREIRA M. S., YANAGIHARA J. I., « A transient three-dimensional heat transfer model of the human body », International Communications in Heat and Mass Transfer, n 36(7), p. 718-724, 2009.

[HEN 14] HenRiQues I. B., MADY C. E. K, OliveiRa JUniOR S., « Obesity and exergy behavior of human body: a first approach ", dans ECOS, June 2014, Turku. Proceedings of the 27th International Conference of Efficiency, Cost, Optimization, Simulation and Environmental Impact of Energy Systems, 2014.

[HER 10] HERSHEY D., Entropy theory of aging systems. Imperial College Press, Londres, 2010.

[IBG 10] IBGE, Pesquisa de orçamentos familiares. Antropometria e estado nutricional de crianças, adolescentes e adultos no Brasil (2008-2009). Ministério da Saúde, Rio de Janeiro, 2010.

[KRO 88] KROMHOUT D., SARIS W. H. M., HORST C. H., « Energy intake, energy expenditure and smoking in relation to body fatness: the Zutphen Study », Am J Clin Nutr, n 47, p. 668-74, 1988.

[MAD 12] Mady C. E. K., Ferreira M. S., Yanagihara J. I., Saldiva P. H. N., Oliveira Junior S., « Modeling the exergy behavior of human body », Energy, n 4(1), p. 546-553, 2012.

[MAD 13a] MADY C. E. K., HENRIQUeS, I. B., OlIVEIRA-JUNIOR S., « Exergy analysis of human body and lifespan: a first approach », dans COBEM, 2013, Ribeirão Preto. Proceedings of the 22th International Congress of Mechanical Engineering, 2013.

[MAD 13b] MADY C. E. K., OLIVEIRA-JUnIOR, S., « Human body exergy metabolism », International Journal of Thermodynamics, $\mathrm{n}^{\circ}$ 16(2), p. 73-80, 2013.

[MOF 91] MofFat R. J., Owens, S. G., « Cessation from cigarette smoking: changes in body weight, body composition, resting metabolism and energy consumption », Metabolism, $\mathrm{n}^{\circ}$ 40(5), p. 465-470, 1991.

[PRI 46] PRIGOGINE I., Wiame J., « Biologie et Thermodynamique des phenomenes irreversibles », Experimentia, ${ }^{\circ}$ 2(11), p. 451-453, 1946.

[RUB 08] RUBNER M., Das Problem der Lebendauer und seine Beziehung zum Wachstum und Ernahnung. Oldenberg, Munich, 1908.

[SCH 92] SCHRODINGER, R., SCHRÖDINGER, E., What is life?: With mind and matter and autobiographical sketches. Cambridge University Press, Cambridge, 1992.

[SIL 09] Silva C., ANNAMALAI K., « Entropy generation and human aging: lifespan entropy and effect of diet composition and caloric restriction diets », Journal of Thermodynamics ${ }^{\circ}$ 2009, p.1-10, 2009.

[SPE 05] SPEAKMAN J. R., « Body size, energy metabolism and lifespan - A review », J. Exp. Biol., n 208, p. 17171730, 2005.

[SZA 05] SZARGUT J. « Exergy Analysis », Research in Progress Thermodynamics, n 7(3), p. 31-33, 2005. 